\title{
Editoriale: perché una rubrica sulla Malattia di Fabry
}

Marco Lombardi

Editor-in-Chief, Giornale di Tecniche Nefrologiche e Dialitiche

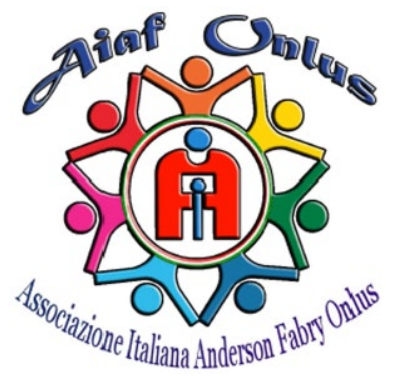

Un detto arcinoto, a proposito di una oramai arcinota malattia infettiva, recita: "se lo conosci lo eviti". 1

Questo aforisma, questo incipit, appena modificato può essere applicato a molte altre patologie e calza assolutamente molto bene per quelle malattie meno conosciute, più rare, $\mathrm{o}$ più difficili da inquadrare perché hanno sintomi in comune con altre malattie assai più frequenti nella popolazione. Ne sono un esempio l'Addison, io stesso l'ho usato in suo prop$\mathrm{osito}^{2}$ ma anche la malattia di Anderson-Fabry (MAF).

La modifica dell'incipit "se lo conosci lo eviti" in "se lo conosci lo riconosci" non è certo di poco conto perché per riconoscere (alias diagnosticare) ovviamente devi conoscere, ovvero devi averne quella conoscenza scientifica auspicata per chi è preposto al nostro lavoro di medico, infermiere, assistente sociale, ecc.

Come Guest Editor del Giornale e come medico da sempre sensibile al coinvolgimento del paziente nelle sue cure ho sentito in questo caso la forte esigenza di mettere il Giornale al servizio di una minoranza silenziosa, che non per questo merita meno di altre più rumorose o note: merita esattamente né più né meno quanto meritano tutti gli altri.

Il Giornale non è nuovo nel mettersi al servizio di minoranze o di casi che sembrano persi ed anche per la MAF si è recentemente adoprato per diffonderne la conoscenza. ${ }^{3}$ Questo servizio che il Giornale propone e che sto qui presentando non significa altro che divenire canale d'informazione e diffusione della conoscenza di questa patologia di cui ancora troppo spesso si ignora l'esistenza, o non la si ricorda più.

Oggi sappiamo che la MAF è effettivamente curabile con successo se presa in tempo utile, perché oggi esistono i mezzi per farlo. Esistono i mezzi per la sua cura, esistono quelli per la prevenzione delle sue complicanze maggiori (tra cui la malattia renale è tra le peggiori assieme a quella cardiaca e neurologica), esistono soprattutto i mezzi per diagnosticarla in tempo. Ed allora non sono più accettabili (almeno da parte di chi scrive) ritardi diagnostici e quindi terapeutici, con quadri clinici che dovranno prima o poi essere appannaggio solo di un passato storico, che non sarà (purtroppo) passato inutilmente, perché sarà stato il principale strumento per indurre il cambiamento attuale che tutti auspichiamo ed aspettiamo.

Ecco il ruolo di questa nuova Rubrica che parte dal 2018, gestita non solo da importanti e riconosciuti cultori della materia ma anche e soprattutto dai tenutari della associazione (AIAF) che rappresenta i pazienti affetti da questa malattia ed i loro famigliari e - cosa importantissima - li riunisce assieme ai cultori deputati al progresso scientifico per la lotta alla MAF. In particolare fanno parte del comitato editoriale di tale rubrica Renzo Mignani (Nefrologo e coordinatore del comitato), Walter Borsini (Neurologo), Giovanni Duro (Genetista), Sandro Feriozzi (Nefrologo), Maurizio Pieroni (Cardiologo), Federico Pieruzzi (Cardiologo) e Stefania Tobaldini (Presidente AIAF).

Saranno proprio i pazienti ed i loro famigliari, assieme a tutti coloro che sono in qualsiasi modo dedicati alla assistenza ai malati di MAF, che ci aiuteranno ad operare la diffusione della conoscenza di questa patologia rara ma che non perdona se presa in ritardo. Sarà parlando sempre di più della MAF, delle sue caratteristiche, delle sue problematiche, delle sue sconfitte, delle paure che genera e finalmente delle nostre vittorie su di 'lei', anche da questo pulpito, che si cercherà di diffondere la conoscenza sulla MAF... perché se la conosci la riconosci e se la riconosci, o prima la riconosci, meglio e più efficacemente la curi, o riesci a ridurne e limitarne la possibilità di nuovi casi... magari con un counseling genetico, psicologico, nefrologico, cardiologico, neurologico, dermatologico, oculistico, ecc: MULTIDISCIPLINARE.

Perché quello che noi ci prefiggiamo anche con questa Rubrica non è cercare di arrivare ad una sua diagnosi nella maggioranza dei casi; no, è viceversa cercare di arrivare al più presto ad una diagnosi appropriata per tempi e modi in tutti i possibili soggetti che ne sono affetti.

Si può fare! Si deve fare! Oggi, subito!

\section{Bibliografia}

1. Pubblicità Progresso AIDS 1991, https://www.youtube. com/watch? $\mathrm{v}=\mathrm{AC} 2 \mathrm{xBwYaJM8}$ (consultato: 8 marzo 2018).

2. Lombardi M, Michelassi S e Betterle C. Conoscerlo per riconoscerlo: morbo di Addison con sindrome poliendocrina autoimmune di Tipo 2. G Tec Nefrol Dial 2013; 1: 37-42.

3. Giovanni Duro e Marco Lombardi, a cura di. La Malattia di Anderson-Fabry. G Tec Nefrol Dial 2017; 29Suppl 1: S1-S36. 